\title{
VARIATIONAL SENSITIVITY ANALYSIS OF PARAMETRIC MARKOVIAN MARKET MODELS
}

\author{
NORBERT HILBER, CHRISTOPH SCHWAB and CHRISTOPH WINTER \\ Seminar for Applied Mathematics, ETH Zürich \\ Rämistrasse 101, 8092 Zürich, Switzerland \\ E-mail: norbert.hilber@sam.math.ethz.ch \\ christoph.schwab@sam.math.ethz.ch \\ christoph.winter@sam.math.ethz.ch
}

\begin{abstract}
Parameter sensitivities of prices for derivative contracts play an important role in model calibration as well as in quantification of model risk. In this paper a unified approach to the efficient numerical computation of all sensitivities for Markovian market models is presented. Variational approximations of the integro-differential equations corresponding to the infinitesimal generators of the market model differentiated with respect to the model parameters are employed. Superconvergent approximations to second and higher derivatives of prices with respect to the price process' state variables are extracted from approximate, computed prices with low, $C^{0}$ regularity by postprocessing. The extracted numerical sensitivities are proved to converge with optimal rates as the mesh width tends to zero. Numerical experiments for uni- and multivariate models with sparse tensor product discretization confirm the theoretical results.
\end{abstract}

1. Introduction. A key task in financial engineering is the fast and accurate calculation of sensitivities of market models with respect to model parameters. This becomes necessary for example in model calibration, risk analysis and in the pricing and hedging of certain derivative contracts. Classical examples are variations of option prices with respect to the spot price or with respect to time-to-maturity, the so-called "Greeks" of the model. For classical, diffusion type models and plain vanilla type contracts, the Greeks can be obtained analytically (see [21]). With the trends to more general market models of jump-diffusion type and to more complicated contracts, closed form solutions are generally not available for pricing and calibration. Thus, prices and model sensitivities have to be approximated numerically. As model sensitivities are generally derivatives of the computed prices with respect to model input parameters, a naive approach consists

2000 Mathematics Subject Classification: Primary 60J25; Secondary 65M60, 65N30.

Key words and phrases: Markov process, Greeks, sensitivity, sparse tensor finite elements. The paper is in final form and no version of it will be published elsewhere. 
in numerically differentiating computed prices by, e.g., Finite Difference formulas. This results in extra work (e.g. due to multiple 'forward' pricing runs) and, due to the generally low regularity of Finite Difference or Finite Element approximations to prices of derivative contracts, in substantial loss of accuracy in the computed sensitivities.

To obtain stable numerical procedures yielding approximate, numerically computable sensitivities for general Markovian market models and for general contracts which converge at the same rate as the computed option prices, additional analytical considerations are necessary.

Most work in this direction has been devoted to Monte-Carlo methods (see [8, 13] and references therein) for diffusion and jump-diffusion models. This paper is focused on a more general class of Markov processes $X$, including stochastic volatility and multidimensional Lévy models. A mesh-based approach is used to solve the corresponding partial integro-differential equation (PIDE). A mesh-based approach is also described in [1] where automatic differentiation of a Finite Element code is used to approximate the Greeks.

In our approach, we distinguish between two classes of sensitivities. The sensitivity of the solution $u$ to variation of a model parameter, like the Greek Vega $\left(\partial_{\sigma} u\right)$ and the sensitivity of the solution $u$ to a variation of state spaces such as the Greek Delta $\left(\partial_{x} u\right)$. We show that an approximation for the first class can be obtained as a solution of the pricing PIDE with a right hand side depending on $u$. For the second class, a finite difference like differentiation procedure is presented which allows to obtain the sensitivities from the Finite Element forward price without additional forward solver.

The outline of the paper is as follows. We start by describing the problem setup. First we explain the abstract framework and the variational discretization of the forward Kolmogorov equation by Finite Element methods. Then, we derive for both classes of sensitivities an algorithm to compute these by postprocessing the Finite Element solution. It is shown that approximation of the sensitivities converge with the same rate as the approximation of the option price. Finally, we give numerical examples for different dimensions and models.

\section{Variational option pricing}

2.1. Parametric Markovian market models. Let $\left(\Omega, \mathcal{F},\left(\mathcal{F}_{t}\right)_{t \geq 0}, \mathbb{P}\right)$ be a filtered probability space satisfying the usual hypotheses. We consider the process $X$ to model the dynamics of a single underlying, a basket or a underlying and its "background" volatility drivers in case of stochastic volatility models. For notational simplicity only we assume that the interest rate is zero. Let $g$ be the payoff, $T>0$ the maturity and $\mathbb{Q}$ an equivalent martingale measure $(\mathrm{EMM})$ to $\mathbb{P}$, i.e. $\mathbb{Q} \sim \mathbb{P}$ such that the process $\left(X_{t}\right)_{t \geq 0}$ is a $\mathbb{Q}$-martingale.

Since $X$ is Markovian, the fair price of a European style contingent claim with underlying $X$ is given by

$$
u(t, x)=\mathbb{E}^{\mathbb{Q}}\left[g\left(X_{T}\right) \mid X_{t}=x\right] .
$$

If the function value $u$ is sufficiently smooth, it is known to solve the backward Kol- 
mogorov equation

$$
\begin{aligned}
-\partial_{t} u+\mathcal{A} u & =0 \quad \text { in }(0, T) \times \mathbb{R}^{d}, \\
u(T, x) & =g(x) \text { in } \mathbb{R}^{d},
\end{aligned}
$$

where $\mathcal{A}$ denotes the infinitesimal generator of $X$. We consider processes $X$ where $\mathcal{A}$ splits into the diffusive part $\mathcal{A}_{W}$, the drift part $\mathcal{A}_{\delta}$ and the jump part $\mathcal{A}_{J}$ given by

$$
\begin{aligned}
\mathcal{A} & =\mathcal{A}_{W}+\mathcal{A}_{\delta}+\mathcal{A}_{J}, \quad \text { where } \\
\mathcal{A}_{W}[\varphi](x) & =-\frac{1}{2} \mathcal{Q}(x) D^{2} \varphi(x), \\
\mathcal{A}_{\delta}[\varphi](x) & =\langle b(x), D \varphi(x)\rangle, \\
\mathcal{A}_{J}[\varphi](x) & =-\int_{E}\left(\varphi(x+\zeta(x, z))-u(x)-\langle\zeta(x, z), D \varphi(x)\rangle 1_{|z| \leq 1}\right) \nu(\mathrm{d} z) .
\end{aligned}
$$

Here, $\mathcal{Q}: \mathbb{R}^{d} \rightarrow \mathbb{R}^{d \times d}, b: \mathbb{R}^{d} \rightarrow \mathbb{R}^{d}$ and $\zeta: \mathbb{R}^{d} \times E \rightarrow \mathbb{R}^{d}$ with the set of admissible jumps $E \subset \mathbb{R}^{d} \backslash\{0\}$. Furthermore, $D$ and $D^{2}$ are the differential operators $D=\left(\partial_{x_{i}}\right)_{1 \leq i \leq d}$, and $D^{2}=\left(\partial_{x_{i} x_{j}}\right)_{1 \leq i, j \leq d}$ and $\nu$ denotes the compensator of a Poisson random measure on $E$ satisfying $\int_{E} \min \left\{1,|z|^{2}\right\} \nu(\mathrm{d} z)<\infty$.

Definition 1 . We call a process $X$ a parametric Markovian market model with admissible parameter set $\mathcal{S}_{\eta}$ if

(i) for all $\eta \in \mathcal{S}_{\eta} X$ is a strong Markov process with respect to a stochastic basis $\left(\Omega, \mathcal{F},\left(\mathcal{F}_{t}\right)_{t \geq 0}, \mathbb{P}\right)$

(ii) the infinitesimal generator $\mathcal{A}$ of the semigroup generated by $X$ has the form (3), and the mapping $\mathcal{S}_{\eta} \ni \eta \rightarrow\{\mathcal{Q}, b, \nu, \zeta\}$ is infinitely differentiable.

In (3), we assume that the coefficients $\mathcal{Q}, b, \zeta$ and $\nu$ do not depend on time $t$. Recently, Carr et al. [10] considered Sato processes (self-similar additive processes) as drivers for the underlying $X$. The authors introduce $\mathbb{R}$-valued pure jump processes with Lévy measure $\nu$ which has time-inhomogeneous Lévy density $k(z, t)$. Our approach to compute prices and sensitivities is not restricted to time independent coefficients but naturally extends to the case when the coefficients are time-inhomogeneous. We give some examples of Markov processes $X$ and their infinitesimal generators covered by our approach.

ExAmple 1 (Multidimensional Lévy model [16, 22]). The Markov process is given by the $d$ dimensional Lévy process $X=\left(X_{1}, \ldots, X_{d}\right)$ with characteristic triplet $\left(\mathcal{Q}, \nu_{\mathbb{Q}}, \gamma\right)$ under the EMM $\mathbb{Q}$. We assume that the Lévy measure satisfies $\int_{|z|>1} e^{z_{i}} \nu_{\mathbb{Q}}(\mathrm{d} z)<\infty$, $i=1, \ldots, d$. Then, the coefficients are given by

$$
\mathcal{Q}(x)=\left(\mathcal{Q}_{i j}\right)_{1 \leq i, j \leq d}, b(x)=\left(\gamma_{i}\right)_{1 \leq i \leq d}, \zeta(x, z)=z,
$$

with $\gamma_{i}=\frac{1}{2} \mathcal{Q}_{i i}+\int_{\mathbb{R}^{d}}\left(e^{z_{i}}-1-z_{i} 1_{|z| \leq 1}\right) \nu(\mathrm{d} z)$. The dependence structure of the Brownian motion part of $X$ is characterized entirely by its covariance matrix $\mathcal{Q}$. The dependence structure of the purely discontinuous part of $X$ can be described using Lévy copulas. These were introduced in Tankov [22] and developed in Kallsen and Tankov [16]. Analytic properties and wavelet discretization of the copula process' generator were discussed by Farkas et al. [11]. For the Clayton Lévy copula with CGMY [9] margins the 
multidimensional Lévy density is given by

$$
k\left(x_{1}, \ldots, x_{d}\right)=\left.\partial_{1} \ldots \partial_{d} F\right|_{\xi_{1}=U_{1}\left(x_{1}\right), \ldots, \xi_{d}=U_{d}\left(x_{d}\right)} k_{1}\left(x_{1}\right) \ldots k_{d}\left(x_{d}\right),
$$

with marginal Lévy densities

$$
k_{i}(z)=C_{i}\left(\frac{e^{G_{i} z}}{|z|^{1+Y_{i}}} 1_{\{z<0\}}+\frac{e^{-M_{i} z}}{|z|^{1+Y_{i}}} 1_{\{z>0\}}\right), \quad C_{i}, G_{i}>0, M_{i}>1, Y_{i}<2,
$$

marginal tail integrals

$$
U_{i}(x)=C_{i} M_{i}^{Y_{i}} \Gamma\left(-Y_{i}, M_{i} x\right) 1_{\{x>0\}}-C_{i} G_{i}^{Y_{i}} \Gamma\left(-Y_{i},-G_{i} x\right) 1_{\{x<0\}},
$$

and Lévy copula

$$
F\left(x_{1}, \ldots, x_{d}\right)=2^{2-d}\left(\sum_{i=1}^{d}\left|x_{i}\right|^{-\theta}\right)^{-\frac{1}{\theta}}\left(\eta 1_{\left\{x_{1} \cdots x_{d} \geq 0\right\}}-(1-\eta) 1_{\left\{x_{1} \cdots x_{d} \leq 0\right\}}\right),
$$

where $i=1, \ldots, d, \theta>0$ and $\eta \in[0,1]$. The Clayton copula density blends for $x_{i} \geq 0$, $i=1, \ldots, d$ the complete independence density $(\theta=0)$ and the complete dependence density $(\theta \rightarrow \infty)$.

EXAmple 2 (Stochastic volatility model of Heston [14]). The Markov process $X$ is of the form $X=(S, Y)$, where the $\mathbb{R}$-valued process $S$ describes the dynamics of the underlying and the $\mathbb{R}$-valued process $Y$ its volatility. Under a EMM $\mathbb{Q}, X$ satisfies the stochastic differential equation $\mathrm{d} X_{t}=b\left(X_{t}\right) \mathrm{d} t+\Sigma\left(X_{t}\right) \mathrm{d} W_{t}$ where $\left(W_{t}\right)$ denotes a two dimensional Brownian motion and the coefficients $b, \Sigma$ are (see [14])

$$
b=\left(\begin{array}{c}
0 \\
\alpha\left(m-Y_{t}\right)-\lambda\left(t, S_{t}, Y_{t}\right)
\end{array}\right), \quad \Sigma=\left(\begin{array}{cc}
\sqrt{Y_{t}} S_{t} & 0 \\
\beta \rho \sqrt{Y_{t}} & \beta \sqrt{1-\rho^{2}} \sqrt{Y_{t}}
\end{array}\right),
$$

with $\alpha>0$ the rate of mean reversion, $m>0$ the long-run mean level of volatility, $\beta \in \mathbb{R}$ and $\rho \in[-1,1]$ the instantaneous correlation. The function $\lambda:[0, T] \times \mathbb{R}_{+} \times \mathbb{R}_{+} \rightarrow \mathbb{R}$ appearing in the second component of the drift $b$ represents the price of volatility and reflects the incompleteness of this market model. The infinitesimal generator $\mathcal{A}$ of $X$ is as in $(3)$ where for $x:=(S, y):=\left(x_{1}, x_{2}\right) \in \mathbb{R}_{+} \times \mathbb{R}_{+}$

$$
b(x)=\left(\begin{array}{c}
0 \\
\alpha\left(m-x_{2}\right)-\lambda\left(t, x_{1}, x_{2}\right)
\end{array}\right), \mathcal{Q}(x)=\Sigma \Sigma^{\top}=\left(\begin{array}{cc}
x_{1}^{2} x_{2} & \beta \rho x_{1} x_{2} \\
\beta \rho x_{1} x_{2} & \beta^{2} x_{2}
\end{array}\right), \nu=0 .
$$

EXAMPLE 3 (Stochastic volatility model of BNS). This stochastic volatility model suggested by Barndorff-Nielsen and Shephard [3] specifies the volatility (of the underlying) as an Ornstein-Uhlenbeck process driven by a Lévy subordinator $L$. The Markov process $X=(S, Y)=\left(e^{Z}, \sigma^{2}\right)$ under an EMM $\mathbb{Q}$ satisfies the SDE (see $\left.[3,19]\right)$

$$
\begin{aligned}
\mathrm{d} Z_{t} & =\left(-\lambda \kappa-\frac{1}{2} \sigma_{t}^{2}\right) \mathrm{d} t+\sigma_{t} \mathrm{~d} W_{t}+\rho \mathrm{d} L_{\lambda t}, \\
\mathrm{~d} \sigma_{t}^{2} & =-\lambda \sigma_{t}^{2} \mathrm{~d} t+\mathrm{d} L_{\lambda t},
\end{aligned}
$$

where $\left(W_{t}\right)$ is a $\mathbb{Q}$-Brownian motion and $\left(L_{\lambda t}\right)$ is a $\mathbb{Q}$-Lévy process. The parameters satisfy $\beta, \mu, \rho, \lambda \in \mathbb{R}, \lambda>0, \rho \leq 0$ and the cumulant transform $\kappa$ is $\kappa(\rho)=\int_{\mathbb{R}_{+}}\left(e^{\rho z}-\right.$ $1) w(z) k(z) \mathrm{d} z$. Here, $w: \mathbb{R}_{+} \rightarrow \mathbb{R}_{+}$satisfies $\int_{\mathbb{R}_{+}}(\sqrt{w(z)}-1)^{2} k(z) \mathrm{d} z<\infty$, and $k$ is the density of the Lévy measure of $L$ under the historical measure $\mathbb{P}$. The infinitesimal 
generator $\mathcal{A}$ of $X$ has the form as in (3), where for $x:=\left(x_{1}, x_{2}\right) \in \mathbb{R} \times \mathbb{R}_{+}$and $z \in \mathbb{R}_{+}$ the coefficients are given by (with $\left.c:=\int_{z \leq 1} z \nu(\mathrm{d} z)\right)$

$$
\begin{gathered}
b(x)=\left(\begin{array}{c}
-\lambda \kappa-\rho c-\frac{1}{2} x_{2} \\
-\lambda x_{2}-c
\end{array}\right), \quad \mathcal{Q}(x)=\left(\begin{array}{cc}
x_{2} & 0 \\
0 & 0
\end{array}\right), \\
\zeta(x, z)=\left(\begin{array}{c}
\rho \\
1
\end{array}\right) z, \quad \nu(\mathrm{d} z)=\lambda w(z) k(z) \mathrm{d} z, \quad E=\mathbb{R}_{+} .
\end{gathered}
$$

Note that the term $\langle\zeta(x, z), D \varphi(x)\rangle 1_{|z| \leq 1}$ appearing in $\mathcal{A}_{J}$ can be omitted here, since $L_{t}$ is a subordinator and hence has sample paths of finite variation.

We calculate the sensitivities of the solution $u$ of (1)-(2) with respect to parameters in the infinitesimal generator $\mathcal{A}$ and with respect to solution arguments $x$ and $t$. We write $\mathcal{A}\left(\eta_{0}\right)$ for a fixed parameter $\eta_{0} \in \mathcal{S}_{\eta}$ to emphasize the dependence of $\mathcal{A}$ on $\eta_{0}$ and change the time to time-to-maturity $t \rightarrow T-t$ in (1)-(2). For sensitivity computation (as well as for domain truncation, cf. [18]), it will be crucial below to admit a non-trivial right hand side. Accordingly, we consider from now on the forward parabolic problem

$$
\begin{aligned}
\partial_{t} u+\mathcal{A}\left(\eta_{0}\right) u & =f & & \text { in }(0, T] \times \mathbb{R}^{d}, \\
u(0, x) & =u_{0} & & \text { in } \mathbb{R}^{d},
\end{aligned}
$$

with $u_{0}=g$. For the numerical implementation we truncate the parabolic PIDE (6)-(7) to a bounded domain $G \subset \mathbb{R}^{d}$ and impose boundary conditions on $\partial G$. Typically, $G$ is a $d$ dimensional hypercube, i.e. $G=\prod_{k=1}^{d}\left(a_{k}, b_{k}\right)$ for some $a_{k}, b_{k} \in \mathbb{R}, b_{k}>a_{k}, k=1, \ldots, d$. We approximate the solution to (6)-(7) by the Finite Element method, which is based on the variational formulation of $(6)-(7)$.

2.2. Variational setting. With a parametric Markovian market model $X$ in the sense of Definition 1 with parameter set $\mathcal{S}_{\eta}$ and infinitesimal generator $\mathcal{A}\left(\eta_{0}\right)$ as in $(3), \eta_{0} \in \mathcal{S}_{\eta}$, we associate to $\mathcal{A}\left(\eta_{0}\right)$ the Dirichlet form $a\left(\eta_{0} ; \cdot, \cdot\right): V \times V \rightarrow \mathbb{R}$ via

$$
a\left(\eta_{0} ; u, v\right):=\left\langle\mathcal{A}\left(\eta_{0}\right) u, v\right\rangle_{V^{*} \times V}, \quad u, v \in V,
$$

with domain $V \stackrel{d}{\hookrightarrow} H$ (dense embedding). We identify $H$ with its dual $H^{*}$ and denote by $V^{*}$ the dual of $V$ so that $V \stackrel{d}{\hookrightarrow} H \cong H^{*} \stackrel{d}{\hookrightarrow} V^{*}$. We denote by $\|\cdot\|,\|\cdot\|_{V}$ the norms in $H, V$, by $(\cdot, \cdot)$ the inner product in $H$ and by $\langle\cdot, \cdot\rangle_{V^{*} \times V}$ the duality pairing between $V$ and its dual $V^{*} \cdot \mathcal{L}(V, W)$ is the vector space of linear and continuous operators $\mathcal{A}: V \rightarrow W$.

We assume $\mathcal{A}\left(\eta_{0}\right) \in \mathcal{L}\left(V, V^{*}\right)$ to be an elliptic, spatial operator given in weak form where the Dirichlet form $a(\cdot ; \cdot, \cdot): \mathcal{S}_{\eta} \times V \times V \rightarrow \mathbb{R}$ satisfies: there exist non-negative constants $\alpha\left(\eta_{0}\right), \beta\left(\eta_{0}\right), \gamma\left(\eta_{0}\right)$ such that

$$
\begin{aligned}
\left|a\left(\eta_{0} ; u, v\right)\right| & \leq \alpha\left(\eta_{0}\right)\|u\|_{V}\|v\|_{V}, \quad \forall u, v \in V, \eta_{0} \in \mathcal{S}_{\eta} \quad \text { (continuity), } \\
a\left(\eta_{0} ; v, v\right) & \geq \beta\left(\eta_{0}\right)\|v\|_{V}^{2}-\gamma\left(\eta_{0}\right)\|v\|_{H}^{2}, \quad \forall v \in V, \eta_{0} \in \mathcal{S}_{\eta} \quad \text { (Gårding inequality). }
\end{aligned}
$$

\section{REMARK 1.}

(i) In general, the space $V$ may depend on the parameter $\eta_{0}$ and we should write $V_{\eta_{0}}$. For notational simplicity, we drop the subscript $\eta_{0}$. 
(ii) We can assume without loss of generality that $\gamma\left(\eta_{0}\right)=0$ in (9) since by the exponential shift $w:=e^{-\gamma\left(\eta_{0}\right) \tau} u$ we obtain $\partial_{t} w+\mathcal{A}\left(\eta_{0}\right) w+\gamma\left(\eta_{0}\right) u=e^{-\gamma\left(\eta_{0}\right) t} f$ and the operator $\mathcal{A}\left(\eta_{0}\right)+\gamma\left(\eta_{0}\right) I$ is coercive on $V$.

Denote by $J$ the time interval $J:=(0, T)$. For $f \in L^{2}\left(J ; V^{*}\right)$ and $u_{0} \in H$ the weak formulation to the problem $(6)-(7)$ is given by:

$$
\begin{aligned}
& \text { Find } u \in L^{2}(J ; V) \cap H^{1}\left(J ; V^{*}\right) \text { such that } \\
& \left(\partial_{t} u(t, \cdot), v\right)+a\left(\eta_{0} ; u(t, \cdot), v\right)=\langle f(t), v\rangle_{V^{*} \times V}, \quad \forall v \in V, \\
& u(0, \cdot)=u_{0} .
\end{aligned}
$$

Under the assumption (8)-(9) the operator $\mathcal{A}\left(\eta_{0}\right)+\gamma\left(\eta_{0}\right) I \in \mathcal{L}\left(V, V^{*}\right)$ defines an isomorphism and (10) admits a unique solution.

We assume that $V$ is Sobolev-type space with smoothness index $r$, i.e.

$$
V=\widetilde{H}^{r}, \quad \widetilde{H}^{0}=H=L^{2} .
$$

Note that $r$ depends on the order of the operator $\mathcal{A}\left(\eta_{0}\right)$. We also assume that the solution $u\left(\eta_{0}\right)$ to (10) has higher regularity in space, $u\left(\eta_{0}\right)(t) \in \mathcal{H}^{s} \subset \widetilde{H}^{r}$ for $t \in(0, T]$, where $\mathcal{H}^{s}$ is again a Sobolev-type space with smoothness index $s$.

ExAmple 4. Consider the multivariate Lévy copula model from Example 1.

(i) It can be shown similar to [18] that $V=\widetilde{H}^{r}(G)$ with $r=1$, if $\mathcal{Q}>0$. Here, for $r \geq 0$, the space $\widetilde{H}^{r}(G)$ is given by $\widetilde{H}^{r}(G)=\left\{\left.u\right|_{G}\left|u \in H^{r}(\mathbb{R}), u\right|_{\mathbb{R} \backslash G}=0\right\}$.

(ii) Now let $\mathcal{Q}_{i j}=b_{i}=0,1 \leq i, j \leq d$, and marginal Lévy densities $k_{i}, 1 \leq i \leq d$, as in (5). In [11] it was proved that for multivariate barrier contracts $V$ is the anisotropic Sobolev space $V=\widetilde{H}^{r}(G)$, with $r=\left(Y_{1} / 2, \ldots, Y_{d} / 2\right)$. Here, for $r=\left(r_{1}, \ldots, r_{d}\right)$, $r_{i} \geq 0, i=1, \ldots, d$, we denote the space $\widetilde{H}^{r}(G)$ by $\widetilde{H}^{r}(G)=\overline{\left\{\bar{u} \mid u \in C_{0}^{\infty}(G)\right\}}$ where $\bar{u}$ is the zero extension of $u$ to $\mathbb{R}^{d}$ and the closure is taken with respect to the norm given by $\|u\|_{H^{r}\left(\mathbb{R}^{d}\right)}^{2}=\sum_{j=1}^{d}\|u\|_{H_{j}^{r_{j}}\left(\mathbb{R}^{d}\right)}^{2}$.

2.3. Variational discretization. Let $V_{h}$ be a finite dimensional subspace $V_{h} \subset V$ consisting of continuous piecewise polynomials of degree $p \geq 1$ with $\operatorname{dim} V_{h}=N<\infty$. The Finite Element semi-discretization in (log) price space of (10) reads:

Find $u_{h} \in L^{2}\left(J ; V_{h}\right) \cap H^{1}\left(J ;\left(V_{h}\right)^{*}\right)$ such that

$$
\begin{aligned}
& \left(\partial_{t} u_{h}(t, \cdot), v_{h}\right)+a\left(\eta_{0} ; u_{h}(t, \cdot), v_{h}\right)=\left\langle f(t), v_{h}\right\rangle_{V^{*} \times V} \quad \forall v_{h} \in V_{h}, \\
& u_{h}(0, \cdot)=u_{0, h},
\end{aligned}
$$

where $u_{0, h}$ is an approximation of $u_{0}$ in $V_{h}$. The Finite Element formulation (12)-(13) is equivalent to a large, but finite system of ODEs to be solved numerically on the time interval $J$. To this end, we fix a basis $\mathcal{B}:=\left\{\Phi_{j}\right\}_{j=1}^{N}$ of $V_{h}$ and let $\underline{u}$ denote the coefficient vector of $u_{h}$ with respect to the basis $\mathcal{B}$. Then, (12)-(13) is equivalent to:

Find $\underline{u}(t) \in \mathbb{R}^{N}$ such that

$$
\mathbf{M} \underline{\dot{u}}+\mathbf{A} \underline{u}=\underline{f}(t),
$$

where $\mathbf{M}$ and $\mathbf{A}$ are the so-called mass and stiffness matrices given by 


$$
\mathbf{M}=\left(\left(\Phi_{i}, \Phi_{j}\right)\right)_{1 \leq i, j \leq N}, \quad \mathbf{A}=\left(a\left(\eta_{0} ; \Phi_{j}, \Phi_{i}\right)\right)_{1 \leq i, j \leq N},
$$

as well as $\underline{f}(t)=\left\langle f(t), \Phi_{j}\right\rangle_{1 \leq j \leq N}$.

For the convergence analysis of the Finite Element based pricing algorithms, we assume the following approximation property of the space $V_{h}$ : For all $u \in \mathcal{H}^{s}$ with $r \leq s \leq p+1$ there exists a $u_{h} \in V_{h}$ such that for $0 \leq \tau \leq r$ (with $r$ as in (11))

$$
\left\|u-u_{h}\right\|_{\widetilde{H}^{\tau}} \leq C h^{s-\tau}\|u\|_{\mathcal{H}^{s}}
$$

We further assume the existence of a projector or an interpolant $P_{h}: V \rightarrow V_{h}$ which satisfies (15) with $u_{h}=P_{h} u$.

We give examples for the space $V_{h}$. In dimension $d=1$, we consider $V_{h}$ to be the wavelet Finite Element space on a uniform mesh with mesh width $h$ on $G$ as proposed e.g. in [18]. In this setting, the projector $P_{h}$ is defined by truncating the wavelet expansion of $u \in V$. For problems in dimension $d \geq 2$, consider the sparse tensor space $\widehat{V}_{h}$ as defined e.g. in $[26]$.

To discretize in time, we use the $\theta$-scheme. For $M \in \mathbb{N}$ define the time step $\Delta t=\frac{T}{M}$ and $t^{m}=m \Delta t, m=0, \ldots, M$. The fully discrete scheme reads: Find $u_{h}^{m+1} \in V_{h}$, $m=0,1, \ldots, M-1$ such that

$$
\left(\Delta t^{-1}\left(u_{h}^{m+1}-u_{h}^{m}\right), v\right)+a\left(\eta_{0} ; u_{h}^{m+\theta}, v_{h}\right)=\left(f^{m+\theta}, v\right), \quad \forall v \in V_{h},
$$

with $u_{h}^{0}=u_{0, h}$. Here $u_{h}^{m+\theta}:=\theta u_{h}^{m+1}+(1-\theta) u_{h}^{m}$ and $f^{m+\theta}:=\theta f\left(t^{m+1}\right)+(1-\theta) f\left(t^{m}\right)$. In matrix form, (16) reads

$$
\left(\Delta t^{-1} \mathbf{M}+\theta \mathbf{A}\right) \underline{u}^{m+1}=\left(\Delta t^{-1} \mathbf{M}-(1-\theta) \mathbf{A}\right) \underline{u}^{m}+\underline{f}^{m+\theta}, \quad m=0,1, \ldots, M-1,
$$

where $\underline{u}^{m}$ is the coefficient vector of $u_{h}^{m}$ with respect to the basis $\mathcal{B}$ of $V_{h}$.

3. Sensitivity analysis. For a parametric Markovian market model $X$ in the sense of Definition 1 we distinguish two classes of sensitivities.

1. The sensitivity of the solution $u$ to a variation $\mathcal{S}_{\eta} \ni \eta_{s}:=\eta_{0}+s \delta \eta, s>0$, of an input parameter $\eta_{0} \in \mathcal{S}_{\eta}$. Typical examples are the Greeks Vega $\left(\partial_{\sigma} u\right)$, Rho $\left(\partial_{r} u\right)$ and Vomma $\left(\partial_{\sigma \sigma} u\right)$. Other sensitivities which are not so commonly used in the financial community are the sensitivity of the price with respect to the jump intensity or the order of the process that models the underlying. We show that the Finite Element approximation to such sensitivities satisfies again the scheme (16) with a right hand side $f^{m+\theta}$ which depends on the approximation $u_{h}^{m+\theta}$ of the pricing function $u$. We also show that the approximation of these sensitivities converge with the same rate as $u_{h}$.

2. The sensitivity of the solution $u$ to a variation of arguments $t, x$. Typical examples are the Greeks Theta $\left(\partial_{\tau} u\right)$, Delta $\left(\partial_{x} u\right)$ and Gamma $\left(\partial_{x x} u\right)$. Higher derivatives like $\partial_{x x x} u$ are used in [12, Chapter 5] to approximate prices of European options under stochastic volatility models. We show that these sensitivities can directly be obtained by postprocessing the Finite Element solution $u_{h}(12)-(13)$ without additional runs. Again our numerical approximations of these sensitivities converge with the same rate as $u_{h}$. 
3.1. Sensitivity with respect to model parameters. Let $\mathcal{C}$ be a Banach space over a domain $G \subset \mathbb{R}^{d} . \mathcal{C}$ is the space of parameters or coefficients in the operator $\mathcal{A}$ and $\mathcal{S}_{\eta} \subseteq \mathcal{C}$ is the set of admissible coefficients. We denote by $u\left(\eta_{0}\right)$ the unique solution to (10) and introduce the derivative of $u\left(\eta_{0}\right)$ with respect to $\eta_{0} \in \mathcal{S}_{\eta}$ as the mapping $D_{\eta_{0}} u\left(\eta_{0}\right): \mathcal{C} \rightarrow V$

$$
\widetilde{u}(\delta \eta):=D_{\eta_{0}} u\left(\eta_{0}\right)(\delta \eta):=\lim _{s \rightarrow 0^{+}} \frac{1}{s}\left(u\left(\eta_{0}+s \delta \eta\right)-u\left(\eta_{0}\right)\right), \quad \delta \eta \in \mathcal{C} .
$$

We also introduce the derivative of $\mathcal{A}\left(\eta_{0}\right)$ with respect to $\eta_{0} \in \mathcal{S}_{\eta}$

$$
\widetilde{\mathcal{A}}(\delta \eta) \varphi:=D_{\eta_{0}} \mathcal{A}\left(\eta_{0}\right)(\delta \eta) \varphi:=\lim _{s \rightarrow 0^{+}} \frac{1}{s}\left(\mathcal{A}\left(\eta_{0}+s \delta \eta\right) \varphi-\mathcal{A}\left(\eta_{0}\right) \varphi\right), \quad \varphi \in V, \quad \delta \eta \in \mathcal{C} .
$$

We assume that $\widetilde{\mathcal{A}}(\delta \eta) \in \mathcal{L}\left(\widetilde{V}, \widetilde{V}^{*}\right)$ with $\widetilde{V}$ a real and separable Hilbert space satisfying

$$
\widetilde{V} \subseteq V \stackrel{d}{\hookrightarrow} H \cong H^{*} \stackrel{d}{\hookrightarrow} V^{*} \subseteq \widetilde{V}^{*}
$$

We further assume that there exists a real and separable Hilbert space $\bar{V} \subseteq \tilde{V}$ such that $\widetilde{\mathcal{A}} v \in V^{*}, \forall v \in \bar{V}$.

We have the following relation between $D_{\eta_{0}} u\left(\eta_{0}\right)(\delta \eta)$ and $u$.

Lemma 1. Let $\widetilde{\mathcal{A}}(\delta \eta) \in \mathcal{L}\left(\widetilde{V}, \widetilde{V}^{*}\right), \forall \delta \eta \in \mathcal{C}$ and $u\left(\eta_{0}\right):(0, T] \rightarrow \bar{V}, \eta_{0} \in \mathcal{S}_{\eta}$ be the unique solution to

$$
\begin{aligned}
\partial_{t} u\left(\eta_{0}\right)+\mathcal{A}\left(\eta_{0}\right) u\left(\eta_{0}\right) & =0 \quad \text { in }(0, T) \times \mathbb{R}^{d}, \\
u\left(\eta_{0}\right)(0, \cdot) & =g(x) \text { in } \mathbb{R}^{d} .
\end{aligned}
$$

Then $\widetilde{u}(\delta \eta)$ solves

$$
\begin{aligned}
\partial_{t} \widetilde{u}(\delta \eta)+\mathcal{A}\left(\eta_{0}\right) \widetilde{u}(\delta \eta) & =-\widetilde{\mathcal{A}}(\delta \eta) u\left(\eta_{0}\right) \quad \text { in }(0, T) \times \mathbb{R}^{d}, \\
\widetilde{u}(\delta \eta)(0, \cdot) & =0 \quad \text { in } \mathbb{R}^{d} .
\end{aligned}
$$

Proof. Since $u\left(\eta_{0}\right)(0)=g$ does not depend on $\eta_{0}$ its derivative with respect to $\eta$ is 0 . Now let $\eta_{s}:=\eta_{0}+s \delta \eta, s>0, \delta \eta \in \mathcal{C}$. Subtract from the equation $\partial_{t} u\left(\eta_{s}\right)(t)+\mathcal{A}\left(\eta_{s}\right) u\left(\eta_{s}\right)(t)=0$ equation (17) and divide by $s$ to obtain

$$
\begin{aligned}
& \partial_{t} \frac{1}{s}\left(u\left(\eta_{s}\right)(t)-u\left(\eta_{0}\right)(t)\right)+\frac{1}{s}\left(\mathcal{A}\left(\eta_{s}\right)-\mathcal{A}\left(\eta_{0}\right)\right) u\left(\eta_{s}\right)(t) \\
& +\frac{1}{s} \mathcal{A}\left(\eta_{0}\right)\left(u\left(\eta_{s}\right)(t)-u\left(\eta_{0}\right)(t)\right)=0 .
\end{aligned}
$$

Taking $\lim _{s \rightarrow 0^{+}}$gives equation (19).

We associate to the operator $-\widetilde{\mathcal{A}}(\delta \eta)$ the Dirichlet form $\widetilde{a}(\delta \eta ; \cdot, \cdot): \widetilde{V} \times \widetilde{V} \rightarrow \mathbb{R}$ which is given by

$$
-\widetilde{a}(\delta \eta ; u, v)=-(\widetilde{\mathcal{A}}(\delta \eta) u, v) .
$$

The variational formulation to $(19)-(20)$ reads: Find $\widetilde{u}(\delta \eta) \in L^{2}(J ; V) \cap H^{1}\left(J ; V^{*}\right)$ such that $\widetilde{u}(\delta \eta)(0, \cdot)=0$ in $H$ and such that

$$
\left(\partial_{t} \widetilde{u}(\delta \eta)(t, \cdot), v\right)+a\left(\eta_{0} ; \widetilde{u}(\delta \eta)(t, \cdot), v\right)=-\widetilde{a}\left(\delta \eta ; u\left(\eta_{0}\right)(t, \cdot), v\right), \quad \forall v \in V .
$$

Note that $(21)$ has a unique solution $\widetilde{u}(\delta \eta) \in V$ due to the assumptions on $a\left(\eta_{0} ; \cdot, \cdot\right), \widetilde{\mathcal{A}}$ and $u\left(\eta_{0}\right) \in \bar{V}$. 
EXAMPLE 5 (CGMY model). We consider a one-dimensional Lévy process $X$ with CGMY density $k$ as in (5). According to (3)-(4), the infinitesimal generator $\mathcal{A}$ has the form

$$
\mathcal{A}[\varphi]=-\frac{1}{2} \sigma^{2} \partial_{x x} \varphi+\left(\frac{1}{2} \sigma^{2}+c\right) \partial_{x} \varphi-\int_{\mathbb{R}}\left\{\varphi(x+z)-\varphi(x)-z 1_{\{|z| \leq 1\}} \partial_{x} \varphi(x)\right\} k(z) \mathrm{d} z
$$

where the constant $c$ depends only on $k$ via $c:=\int_{\mathbb{R}}\left(e^{z}-1-z 1_{\{|z| \leq 1\}}\right) k(z) \mathrm{d} z$ to ensure that $e^{X}$ is a martingale. The weak formulation for the price of European style contingent claim is as in (10) with $f=0$.

For the sensitivity of the price with respect to the volatility $\sigma$ the set of admissible parameters $\mathcal{S}_{\eta}$ is $\mathcal{S}_{\eta}=\mathbb{R}_{+}$with $\eta=\sigma$. We have

$$
\widetilde{\mathcal{A}}(\delta \sigma) \varphi=-\delta \sigma \sigma_{0} \partial_{x x} \varphi+\delta \sigma \sigma_{0} \partial_{x} \varphi \in \mathcal{L}\left(V, V^{*}\right),
$$

with $\delta \sigma \in \mathbb{R}=\mathcal{C}$. The Dirichlet form $\widetilde{a}(\delta \sigma ; \cdot, \cdot)$ appearing in the weak formulation (21) of $\widetilde{u}(\delta \sigma)$ is given by

$$
\widetilde{a}(\delta \sigma ; \varphi, \psi)=\delta \sigma \sigma_{0}\left(\partial_{x} \varphi, \partial_{x} \psi\right)+\delta \sigma \sigma_{0}\left(\partial_{x} \varphi, \psi\right) .
$$

For the sensitivity of the price with respect to the jump intensity parameter $Y$ of the Lévy process $X$ we let $0<Y<2$. Then, we have $\mathcal{S}_{\eta}=(0,2)$ with $\eta=Y$ and

$$
\widetilde{\mathcal{A}}(\delta Y) \varphi=-\delta Y \int_{\mathbb{R}}\left\{\varphi(x+z)-\varphi(x)-z \partial_{x} \varphi(x)\right\} \widetilde{k}(z) \mathrm{d} z \in \mathcal{L}\left(\widetilde{V}, \widetilde{V}^{*}\right)
$$

where the kernel $\widetilde{k}$ is given by

$$
\widetilde{k}(z):=-\ln |z| k(z) .
$$

It is easy to check that due to $Y<2$ in (5)

$$
\int_{|z| \leq 1} z^{2} \widetilde{k}(z) \mathrm{d} z<\infty, \quad \int_{|z|>1} \widetilde{k}(z) \mathrm{d} z<\infty .
$$

In this setting, $\widetilde{V}=V=\widetilde{H}^{1}(G)$, if $\sigma>0$, and $\widetilde{V}=\widetilde{H}^{Y / 2+\varepsilon}(G) \subset \widetilde{H}^{Y / 2}(G)=V, \forall \varepsilon>0$, if $\sigma=0$ and if the drift has been removed by a change of variables as in [18].

The fully discrete scheme to find an approximation to $\widetilde{u}(\delta \eta)$ in $(21)$ is:

Given $\widetilde{u}_{h}^{0}=0$, for $m=0,1, \ldots, M-1$ find $\widetilde{u}_{h}^{m+1} \in V_{h}$ such that

$$
\left(\Delta t^{-1}\left(\widetilde{u}_{h}^{m+1}-\widetilde{u}_{h}^{m}\right), v\right)+a\left(\eta_{0} ; \widetilde{u}_{h}^{m+\theta}, v_{h}\right)=-\widetilde{a}\left(\delta \eta ; u_{h}^{m+\theta}, v\right), \quad \forall v \in V_{h}
$$

or in matrix form

$$
\left(\Delta t^{-1} \mathbf{M}+\theta \mathbf{A}\right) \underline{\widetilde{u}}^{m+1}=\left(\Delta t^{-1} \mathbf{M}-(1-\theta) \mathbf{A}\right) \underline{\widetilde{u}}^{m}-\widetilde{\mathbf{A}}\left(\theta \underline{u}^{m+1}+(1-\theta) \underline{u}^{m}\right),
$$

where $\widetilde{\mathbf{A}}$ is the matrix of the Dirichlet form $\widetilde{a}(\delta \eta ; \cdot, \cdot)$ in the basis $\mathcal{B}$,

$$
\widetilde{\mathbf{A}}=\left(\widetilde{a}\left(\delta \eta ; \Phi_{j}, \Phi_{i}\right)\right)_{1 \leq i, j \leq N} .
$$

The resulting algorithm is illustrated as pseudo code in Table 1. Here, we denote by $\underline{y} \leftarrow \operatorname{solve}(\mathbf{B}, \underline{x})$ the output of a generic solver for a linear system $\mathbf{B} \underline{x}=\underline{y}$. 
Choose $\eta_{0} \in \mathcal{S}_{\eta}, \delta \eta \in \mathcal{C}$.

Calculate the matrices $\mathbf{M}, \mathbf{A}$ and $\widetilde{\mathbf{A}}$ according to (14) and (23).

Let $\underline{u}^{0}$ be the coefficient vector of $u_{h}^{0}$ in the basis $\mathcal{B}$ of $V_{h}$.

Set $\underline{\widetilde{u}}^{0}=\underline{0}$.

For $j=0,1, \ldots, M-1$

$\underline{u}^{1} \leftarrow \operatorname{solve}\left(\Delta t^{-1} \mathbf{M}+\theta \mathbf{A},\left(\Delta t^{-1} \mathbf{M}-(1-\theta) \mathbf{A}\right) \underline{u}^{0}\right)$

Set $f:=\widetilde{\mathbf{A}}\left(\theta \underline{u}^{1}+(1-\theta) \underline{u}^{0}\right)$

$\left.\underline{\tilde{u}}^{1} \leftarrow \operatorname{solve}\left(\Delta t^{-1} \mathbf{M}+\theta \mathbf{A},\left(\Delta t^{-1} \mathbf{M}-(1-\theta) \mathbf{A}\right) \underline{\tilde{u}}^{0}-\underline{f}\right)\right)$

Set $\underline{u}^{0}:=\underline{u}^{1}, \underline{\widetilde{u}}^{0}:=\underline{u}^{1}$

Next $j$

Table 1. Algorithm to compute sensitivities with respect to model parameters

3.2. Convergence rates for sensitivities with respect to model parameters. In this section we establish convergence rates for the sequence $\left\{\underline{\widetilde{u}}^{m}\right\}_{m=0}^{M-1}$ of sensitivities with respect to model parameters as the discretization parameter $h$ in (12)-(13) tends to zero. We show that the computed sensitivities converge essentially at the same rate as the computed prices. For notational simplicity the subscript $\eta_{0}$ is omitted. We define the energy norm

$$
\|u\|_{a}:=\sqrt{a(u, u)} \sim\|u\|_{V}
$$

which is, by (8) and (9), equivalent to the norm $\|\cdot\|_{V}$. For $f \in V_{h}^{*}$, we let

$$
\|f\|_{*}:=\sup _{0 \neq v_{h} \in V_{h}} \frac{\left(f, v_{h}\right)}{\left\|v_{h}\right\|_{a}} .
$$

The main result of this section is the following Theorem. The proof is given in Appendix A.1.

Theorem 2. Let the assumptions of Lemma A.5 and Lemma A.1 be fulfilled. Then

$$
\begin{aligned}
& \left\|\widetilde{u}^{M}-\widetilde{u}_{h}^{M}\right\|^{2}+\Delta t \sum_{m=0}^{M-1}\left\|\widetilde{u}^{m+\theta}-\widetilde{u}_{h}^{m+\theta}\right\|_{V}^{2} \\
& \leq C \sum_{v \in\{u, \widetilde{u}\}}\left\{\begin{array}{ll}
(\Delta t)^{2} \int_{0}^{T}\|\ddot{v}(\tau)\|_{*}^{2} \mathrm{~d} \tau & \theta \in[0,1] \\
(\Delta t)^{4} \int_{0}^{T}\|\dddot{v}(\tau)\|_{*}^{2} \mathrm{~d} \tau & \theta=\frac{1}{2}
\end{array}+C h^{2(s-r)} \sum_{v \in\{u, \widetilde{u}\}} \int_{0}^{T}\|\dot{v}(\tau)\|_{\mathcal{H}^{s-r}}^{2} \mathrm{~d} \tau\right. \\
& +C h^{2(s-r)} \max _{0 \leq t \leq T}\|u(t)\|_{\mathcal{H}^{s}}^{2} .
\end{aligned}
$$

Theorem 2 shows that if the error between the exact and the approximate price satisfies $\left\|u^{m}-u_{h}^{m}\right\|=O\left(h^{s-r}\right)+O\left((\Delta t)^{\kappa}\right)$, the error between the exact and approximate sensitivity preserves the same convergence rates both in space and time, i.e. $\left\|\widetilde{u}^{m}-\widetilde{u}_{h}^{m}\right\|=$ $O\left(h^{s-r}\right)+O\left((\Delta t)^{\kappa}\right)$.

3.3. Sensitivity with respect to solution arguments. Let $u$ be the solution of the vari-

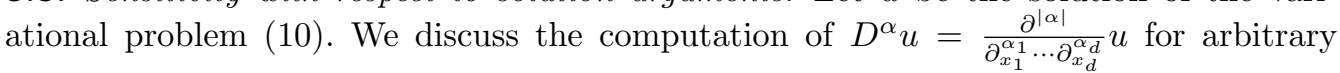
multi-index $\alpha \in \mathbb{N}_{0}^{d}$. The approximation of derivatives of solutions to elliptic and parabolic partial differential equations in the context of Finite Elements is well studied $[2,5,6$, 
$7,20,23,24]$. In these papers, derivatives are approximated by applying difference operators or local averaging operators to the Finite Element solution of the problem. We follow this approach. For $\mu \in \mathbb{Z}^{d}$ a multi-integer and $h \in \mathbb{R}_{+}$we define the translation operator $T_{h}^{\mu} \varphi(x)=\varphi(x+\mu h)$ and the forward difference quotient $\partial_{h, j} \varphi(x)=h^{-1}\left(T_{h}^{e_{j}} \varphi(x)-\varphi(x)\right)$, where $e_{j}, j=1, \ldots, d$, denotes the $j$-th standard basis vector in $\mathbb{R}^{d}$. For $\alpha \in \mathbb{N}_{0}^{d}$ we denote $\partial_{h}^{\alpha} \varphi=\partial_{h, 1}^{\alpha_{1}} \cdots \partial_{h, d}^{\alpha_{d}} \varphi$ and by $D_{h}^{\alpha}$ the difference operator of order $n \geq 0$

$$
D_{h}^{\alpha} \varphi:=\sum_{\gamma,|\alpha|=n} C_{\gamma, \alpha} T_{h}^{\gamma} \partial_{h}^{\alpha} \varphi
$$

Definition 2. The difference operator $D_{h}^{\alpha}$ of order $|\alpha|=n$ and mesh width $h$ is called an approximation to the derivative $D^{\alpha}$ of order $s \in \mathbb{N}_{0}$ if for any $G_{0} \subset G$,

$$
\left\|D^{\alpha} \varphi-D_{h}^{\alpha} \varphi\right\|_{\widetilde{H}^{r}\left(G_{0}\right)} \leq C h^{s}\|\varphi\|_{\mathcal{H}^{s+r+n}(G)}, \quad \forall \varphi \in \mathcal{H}^{s+r+n} .
$$

Given a basis $\mathcal{B}:=\left\{\Phi_{j}\right\}_{j=1}^{N}$ of $V_{h}$, the action of $D_{h}^{\alpha}$ to $v_{h} \in V_{h}$ can be realized as matrix-vector multiplication $\underline{v}_{h} \mapsto \mathbf{D}_{h}^{\alpha} \underline{v}_{h}$, where

$$
\mathbf{D}_{h}^{\alpha}=\left(D_{h}^{\alpha} \Phi_{1}, \ldots, D_{h}^{\alpha} \Phi_{N}\right) \in \mathbb{R}^{N \times N}
$$

and $\underline{v}_{h}$ is the coefficient vector of $v_{h}$ with respect to the basis $\mathcal{B}$.

EXAmPLE 6. Let $\left.\mathcal{V}_{h}=\operatorname{span}\left\{\varphi_{j}(x) \mid 1 \leq j \leq N\right\}, \varphi_{j}(x)=\max \left\{0,1-h^{-1} \mid x-j h\right) \mid\right\}$, $h=\frac{1}{N+1}$, be the space of piecewise linear continuous functions on $[0,1]$ vanishing at the end points 0 , 1 . For $\alpha, \beta, \gamma \in \mathbb{R}$ and $\mu \in \mathbb{N}_{0}$ we denote by $\operatorname{diag}_{ \pm \mu}(\alpha, \beta, \gamma)$ the matrices

$$
\begin{aligned}
& \operatorname{diag}_{-\mu}(\alpha, \beta, \gamma)=\left(\begin{array}{ccccccc}
\cdots & 0 & \alpha & \beta & \gamma & 0 & \cdots \\
& & & \alpha & \beta & \gamma & \\
& & & & \ddots & \ddots & \ddots
\end{array}\right) \\
& \begin{array}{c}
\uparrow \\
\mu+1 \text {-th column }
\end{array}
\end{aligned}
$$

and $\operatorname{diag}_{\mu}(\alpha, \beta, \gamma)=\left(\operatorname{diag}_{-\mu}(\alpha, \beta, \gamma)\right)^{\top}$. Then the matrices $\mathbf{Q}_{h}$ of the forward difference quotient $\partial_{h}$ and $\mathbf{T}_{\mu}$ of the translation operator $T_{h}^{\mu}$ are given by

$$
\mathbf{Q}_{h}=h^{-1} \operatorname{diag}_{0}(0,-1,1), \quad \mathbf{T}_{\mu}=\operatorname{diag}_{\mu}(0,1,0)
$$

Hence, for example, we have for the centered finite difference quotient

$$
D_{h}^{2} \varphi(x)=h^{-2}(\varphi(x+h)-2 \varphi(x)+\varphi(x-h))
$$

of order 2 in one dimension $\mathbf{D}_{h}^{2}=\mathbf{T}_{-1} \mathbf{Q}_{h}^{2}=h^{-2} \operatorname{diag}_{0}(1,-2,1)$.

Now let $V_{h}=\mathcal{V}_{h} \otimes \cdots \otimes \mathcal{V}_{h}$ be the $d$-fold tensor product of $\mathcal{V}_{h}$. Then the matrix $\mathbf{D}_{h}^{\alpha}$ is given by

$$
\mathbf{D}_{h}^{\alpha}=\sum_{\gamma,|\alpha|=n} C_{\gamma, \alpha} \mathbf{T}_{\gamma_{1}} \otimes \cdots \otimes \mathbf{T}_{\gamma_{d}} \mathbf{Q}_{h}^{\alpha_{1}} \otimes \cdots \otimes \mathbf{Q}_{h}^{\alpha_{d}} .
$$

In Table 2 the algorithm to obtain an approximation to the derivative $D^{\alpha} u(T, x)$ at maturity $T$ is illustrated. The vector $\underline{v} \in \mathbb{R}^{N}$ is the coefficient vector of $D_{h}^{\alpha} u_{h}^{M}$ in the basis $\mathcal{B}$ of $V_{h}$. 
Choose $\eta_{0} \in \mathcal{S}_{\eta}$.

Calculate the matrices $\mathbf{M}, \mathbf{A}$ and $\mathbf{D}_{h}^{\alpha}$ according to (14) and (25).

Let $\underline{u}^{0}$ be the coefficient vector of $u_{h}^{0}$ in the basis $\mathcal{B}$ of $V_{h}$.

For $j=0,1, \ldots, M-1$

$$
\begin{aligned}
& \underline{u}^{1} \leftarrow \operatorname{solve}\left(\Delta t^{-1} \mathbf{M}+\theta \mathbf{A},\left(\Delta t^{-1} \mathbf{M}-(1-\theta) \mathbf{A}\right) \underline{u}^{0}\right) \\
& \text { Set } \underline{u}^{0}:=\underline{u}^{1}
\end{aligned}
$$

Next $j$

Set $\underline{v}:=\mathbf{D}_{h}^{\alpha} \underline{u}^{1}$

Table 2. Algorithm to compute with respect to arguments of solution

3.4. Convergence rates for sensitivities with respect to solution arguments. For simplicity, we shall assume in this section that the function $\zeta: \mathbb{R}^{d} \times E \rightarrow \mathbb{R}^{d}$ appearing in (3) depends only on $z$, i.e. $\zeta: E \rightarrow \mathbb{R}^{d}$.

We have the following convergence result for the approximation of sensitivities with respect to solution arguments. Its proof can be found in the Appendix A2.

TheOREm 3. Let the assumptions of Lemma A.1 be fulfilled and assume that $u(x, t)$ is sufficiently smooth in $[0, T] \times \bar{G}$. Assume that the approximation $\partial_{h}^{\beta} u_{h}^{0}$ is quasi-optimal in $L^{2}(G)$ for all $\beta \leq \alpha$. Assume further that $D_{h}^{\alpha}$ approximates $D^{\alpha}$ in the sense of Definition 2. Then

$$
\begin{aligned}
& \left\|D^{\alpha} u^{M}-D_{h}^{\alpha} u_{h}^{M}\right\|^{2}+\Delta t \sum_{m=0}^{M-1}\left\|D^{\alpha} u^{m+\theta}-D_{h}^{\alpha} u_{h}^{m+\theta}\right\|_{V}^{2} \\
& \leq C\left\{\begin{array}{c}
(\Delta t)^{2} \int_{0}^{T}\|\ddot{u}(\tau)\|_{*}^{2} \mathrm{~d} \tau, \quad \theta \in[0,1] \\
(\Delta t)^{4} \int_{0}^{T}\|\dddot{u}(\tau)\|_{*}^{2} \mathrm{~d} \tau, \quad \theta=\frac{1}{2}
\end{array}+C h^{2(s-r)} \int_{0}^{T}\|\dot{u}(\tau)\|_{\mathcal{H}^{s-r}}^{2} \mathrm{~d} \tau\right. \\
& +C h^{2(s-r)} \max _{0 \leq t \leq T}\|u(t)\|_{\mathcal{H}^{s}}^{2} .
\end{aligned}
$$

REMARK 2 .

(i) Note that we cannot get higher convergence rates than $s-r$, even if $u$ has higher regularity $\left(u(t) \in \mathcal{H}^{s+r+n}\right)$.

(ii) Theorem 3 shows that arbitrary derivatives of $u$ can be approximated with the same rate as $u$ itself, provided $u$ is sufficiently smooth.

4. Numerical examples. In this section we compute various sensitivities for different models. We mainly choose models where the price is known in closed form such that we are able to compute the errors between the exact price/sensitivities and their Finite Element approximations. In Theorems 2 and 3 these discretization errors are estimated in the energy norm. In the numerical examples, however, we measure the errors in the $L^{\infty}$ norm since the above mentioned closed form solutions are not given explicitly as functions of $x$ and $t$. This makes the computation of $\left\|u(T, x)-u_{h}^{M}\right\|_{V}$ very expensive and only approximative.

We measure the $L^{\infty}$ norm of the error on a subset $G_{0}$ of the computational domain $G$ at time to maturity $t=T$. In all computations, we choose wavelet Finite Element spaces spanned by continuous wavelets of polynomial degree $p=1$. For problems in dimension 
$d \geq 2$, we choose the sparse grid spaces $\widehat{V}_{h}$ to reduce the computational complexity of the approximations. In the $\theta$-scheme, we let $\theta=\frac{1}{2}$ and choose the time step $\Delta t$ sufficiently small.

The experimental convergence rates are obtained by the least square method applied to the data $\left(\log h, \log e_{h}\right)$, where $e_{h}:=\left\|u(T, x)-u_{h}^{M}\right\|_{L^{\infty}\left(G_{0}\right)}$.

4.1. One-dimensional models. We consider Example 1 with $d=1$ for two models: (i) the Black-Scholes model [4] and (ii) Variance Gamma model [17] with parameters $(\sigma, \nu, \vartheta)$, i.e one has in (5) $Y=0, C=\nu^{-1}, M=\left(\nu \mu_{+}\right)^{-1}$ and $G=\left(\nu \mu_{+}\right)^{-1}$ where $\mu_{+}=$ $\frac{1}{2} \sqrt{\vartheta^{2}+2 \sigma^{2} \nu^{-1}}+\frac{\vartheta}{2}, \mu_{-}=\mu_{+}-\vartheta$. For both models, we consider a European put with strike $K=1$ and maturity $T=0.1$, and we calculate the Greeks Delta, $\Delta=D u=\frac{\partial u}{\partial S}$, and Gamma, $\Gamma=D^{2} u=\frac{\partial^{2} u}{\partial S^{2}}$. For the Black-Scholes model we additionally compute the Vega, $\mathcal{V}=\widetilde{u}(\delta \sigma)=\frac{\partial u}{\partial \sigma}$. We choose for both models the parameter $\sigma=0.4$ as well as $\nu=0.04, \vartheta=-0.2$ for (ii). Using the analytic solution for the Black-Scholes model [4] and the Variance Gamma model [17], we can compute the error of the Finite Element solution. The convergence rates in $\|\cdot\|_{L^{\infty}([0.5,2])}$ are shown in Figure 1.
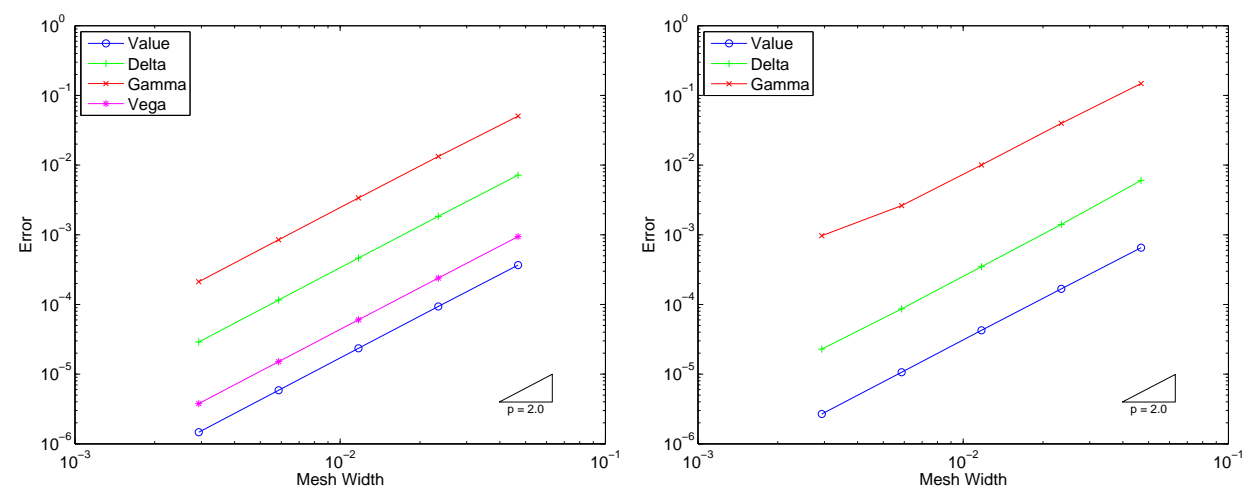

Fig. 1. Convergence rates of Greeks for a European put in the Black-Scholes (left) and Variance Gamma (right) model

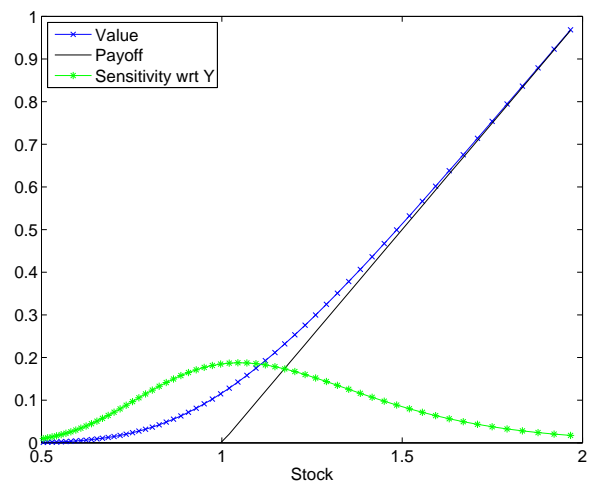

Fig. 2. Computed sensitivity of a European call with respect to the jump intensity parameter $Y$ in the CGMY model 
As predicted in Theorem 2 and 3, all Greeks convergence with the same rate as the price $u$ itself. Here, since the degree of polynomials in $V_{h}$ is $p=1$, the rate is $s=p+1=2$, even when the error is measured in the $L^{\infty}$-norm.

As a further illustration of the method, we compute the sensitivity with respect to the order $Y \widetilde{u}(\delta Y)=\frac{\partial u}{\partial Y}$ as explained in Example 5. We consider a European call with strike $K=1$ and maturity $T=0.5$ and choose the model parameters $C=1, G=12$, $M=10$ and $\eta_{0}=Y=1$. The functions $u(T, S)$ and $\widetilde{u}(\delta Y)(T, S)$ for $\delta Y=1$ are shown in Figure 2.

4.2. Multi-variate models. We consider the Heston stochastic volatility model and a three dimensional basket option.

4.2.1. Heston model. We calculate the sensitivities $\widetilde{u}(\delta \rho)$ and $\widetilde{u}(\delta \alpha)$ with respect to correlation $\rho$ of the Brownian motions that drive the underlying and the volatility and the rate of mean reversion $\alpha$ (see Example 2). To this end, we consider a European call with strike $K=1$ and maturity $T=\frac{1}{2}$. The model parameters for both sensitivity runs are $\lambda=0, \sigma=0.5$ and $m=0.06$. Additionally, for the sensitivity with respect to $\rho$ we let $\rho_{0}=-0.5, \delta \rho=1$ and $\alpha=2.5$. For the sensitivity with respect to $\alpha$ we set $\alpha_{0}=2.5$, $\delta \alpha=1$ and $\rho=-0.5$. We compare the Finite Element solution with the closed form solution given in [14]. The convergence rates in $\|\cdot\|_{L^{\infty}\left(\left[e^{-0.25}, e^{0.75}\right] \times[0.24,1.2]\right)}$ are shown in Figure 3 .

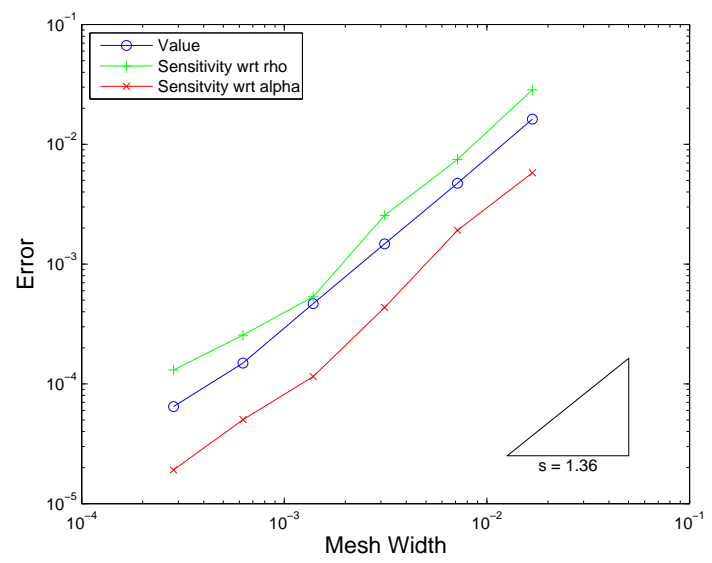

Fig. 3. Convergence rates of sensitivities $\widetilde{u}(\delta \rho), \widetilde{u}(\delta \alpha)$ for a European call in the Heston stochastic volatility model

The experimental convergence rate $s$ is $s \approx 1.36$ for $u$ and $\widetilde{u}(\delta \rho)$ and $s \approx 1.43$ for $\widetilde{u}(\delta \alpha)$. This confirms the theoretical finding of Theorem 2 that computed prices and sensitivities converge with the same rate. In Figure 4 the sensitivities with respect to $\rho$ and $\alpha$ are shown. 

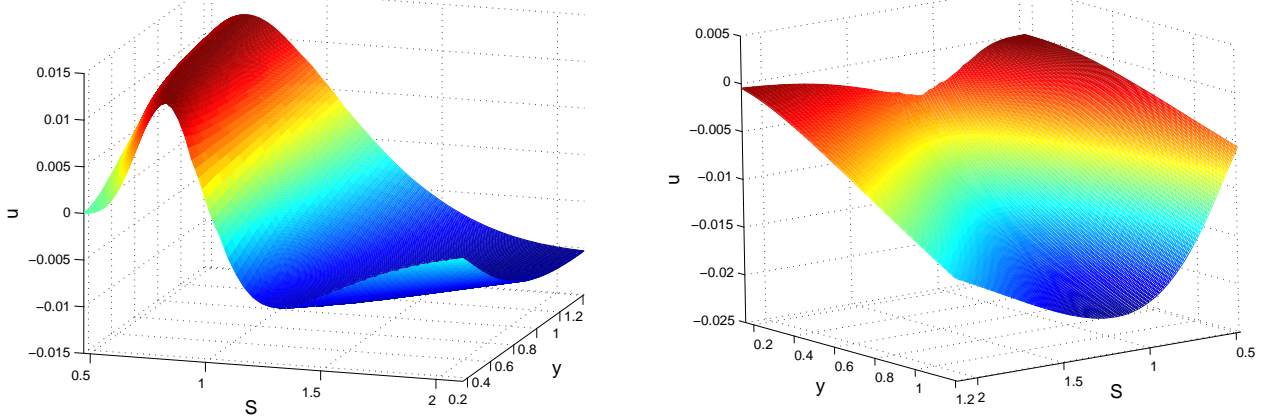

Fig. 4. Computed sensitivities for a European call with respect to model parameters $\rho$ and $\alpha$ : $\widetilde{u}(\delta \rho)$ (left) and $\widetilde{u}(\delta \alpha)$ (right) in the Heston stochastic volatility model

4.2.2. Basket option. We again need an analytic solution to compare our Finite Element solution with. Therefore, we choose $g(S)=\left(\prod_{i=1}^{d} S_{i}-K\right)^{+}$where for the multidimensional Black-Scholes model analytic solutions can be found by reducing the problem to a one-dimensional problem [15, Section 7.5]. We consider the dimension $d=3$, strike $K=1$ and maturity $T=0.1$, and we calculate the Greeks Delta, $\Delta_{1}=\frac{\partial u}{\partial S_{1}}$, and Gamma, $\Gamma_{11}=\frac{\partial^{2} u}{\partial S_{1}^{2}}$. The parameters are $\sigma=0.4$ and $\rho=0$. The convergence rates in $\|\cdot\|_{L^{\infty}\left([0.5,2]^{3}\right)}$ are shown in Figure 5.

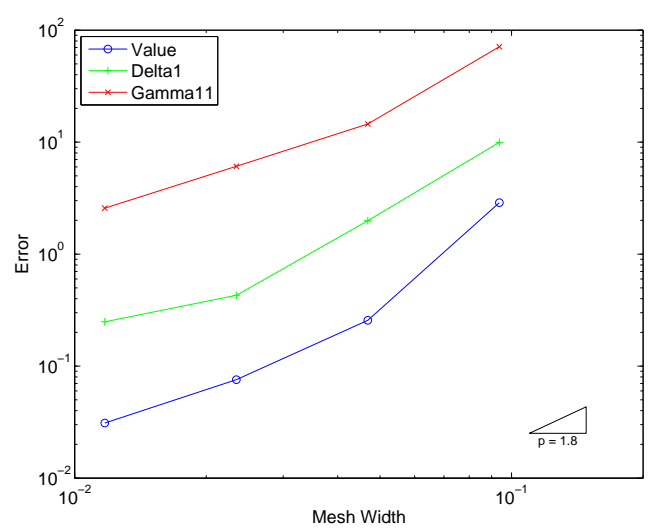

Fig. 5. Convergence rates of Greeks of a basket option in a three dimensional Black-Scholes model

The experimental convergence rate $s$ is $s \approx 1.82$ for $\Delta_{1}$ and $s \approx 1.56$ for $\Gamma_{11}$.

\section{A. Appendix}

A.1. Proof of Theorem 2. We first recall the following stability result for the $\theta$-scheme from [25, Proposition 4.1]. We denote by $\lambda$ the constant

$$
\lambda:=\sup _{0 \neq v_{h} \in V_{h}} \frac{\left\|v_{h}\right\|^{2}}{\left\|v_{h}\right\|_{*}^{2}} .
$$


Lemma A.1. If $\frac{1}{2} \leq \theta \leq 1$, assume that $0<C_{1}<2, C_{2} \geq\left(2-C_{1}\right)^{-1}$. If $0 \leq \theta<\frac{1}{2}$ assume that

$$
\sigma:=\Delta t(1-2 \theta) \lambda<2, \quad 0<C_{1}<2-\sigma, \quad C_{2} \geq \frac{1+\left(4-C_{1}\right) \sigma}{2-\sigma-C_{1}} .
$$

Then the sequence $\left\{u_{h}^{m}\right\}_{m=0}^{M}$ generated by the $\theta$-scheme (16) satisfies the stability estimate

$$
\left\|u_{h}^{M}\right\|^{2}+C_{1} \Delta t \sum_{m=0}^{M-1}\left\|u_{h}^{m+\theta}\right\|_{a}^{2} \leq\left\|u_{h}^{0}\right\|^{2}+C_{2} \Delta t \sum_{m=0}^{M-1}\left\|f^{m+\theta}\right\|_{*}^{2} .
$$

We will also need the following convergence result proved in [25, Theorem 5.4].

Lemma A.2. Let the assumptions of Lemma A.1 be fulfilled. Assume that $u(x, t)$ is sufficiently smooth in $[0, T] \times \bar{G}$ and assume that the approximation property (15) holds. Assume further that the approximation $u_{0, h}=u_{h}^{0} \in V_{h}$ of $u_{0}$ is quasi-optimal in $L^{2}(G)$. Then the sequence $\left\{u_{h}^{m}\right\}_{m=0}^{M-1}$ in (16) satisfies

$$
\begin{aligned}
& \left\|u^{M}-u_{h}^{M}\right\|^{2}+\Delta t \sum_{m=0}^{M-1}\left\|u^{m+\theta}-u_{h}^{m+\theta}\right\|_{V}^{2} \\
& \leq C\left\{\begin{array}{c}
(\Delta t)^{2} \int_{0}^{T}\|\ddot{u}(\tau)\|_{*}^{2} \mathrm{~d} \tau, \quad \theta \in[0,1] \\
(\Delta t)^{4} \int_{0}^{T}\|\dddot{u}(\tau)\|_{*}^{2} \mathrm{~d} \tau, \quad \theta=\frac{1}{2}
\end{array}+C h^{2(s-r)} \max _{0 \leq t \leq T}\|u(t)\|_{\mathcal{H}^{s}}^{2}\right. \\
& \quad+C h^{2(s-r)} \int_{0}^{T}\|\dot{u}(\tau)\|_{\mathcal{H}^{s-r}}^{2} \mathrm{~d} \tau .
\end{aligned}
$$

We now estimate the error $e_{h}^{m}:=\widetilde{u}^{m}-\widetilde{u}_{h}^{m}$, where we set $\widetilde{u}^{m}:=\widetilde{u}\left(t^{m}\right)$. We write

$$
e_{h}^{m}=\underbrace{\widetilde{u}^{m}-P_{h} \widetilde{u}^{m}}_{\eta^{m}}+\underbrace{P_{h} \widetilde{u}^{m}-\widetilde{u}_{h}^{m}}_{\xi_{h}^{m}}=\eta^{m}+\xi_{h}^{m} .
$$

Since $\eta^{m}$ can be estimated by the approximation property (15), we now focus on $\xi_{h}^{m}$.

Lemma A.3. Assume $\widetilde{u} \in C^{1}([0, T] ; H)$. Then $\left\{\xi_{h}^{m}\right\}_{m=0}^{M-1}$ satisfy the $\theta$-scheme: Given $\xi_{h}^{0}=0$, for $m=0,1, \ldots, M-1$

$$
(\Delta t)^{-1}\left(\xi_{h}^{m+1}-\xi_{h}^{m}, v_{h}\right)+a\left(\xi_{h}^{m+\theta}, v_{h}\right)=\left(r^{m}, v_{h}\right), \quad \forall v_{h} \in V_{h}
$$

with weak residual $r^{m}: V_{h} \rightarrow \mathbb{R}$ given by $r^{m}=\sum_{j=1}^{4} r_{j}^{m}$ where

$$
\begin{aligned}
& \left(r_{1}^{m}, v_{h}\right):=\left((\Delta t)^{-1}\left(\widetilde{u}^{m+1}-\widetilde{u}^{m}\right)-\dot{\vec{u}}^{m+\theta}, v_{h}\right), \\
& \left(r_{2}^{m}, v_{h}\right):=\left((\Delta t)^{-1}\left(P_{h} \widetilde{u}^{m+1}-P_{h} \widetilde{u}^{m}\right)-(\Delta t)^{-1}\left(\widetilde{u}^{m+1}-\widetilde{u}^{m}\right), v_{h}\right), \\
& \left(r_{3}^{m}, v_{h}\right):=a\left(P_{h} \widetilde{u}^{m+\theta}-\widetilde{u}^{m+\theta}, v_{h}\right), \\
& \left(r_{4}^{m}, v_{h}\right):=\widetilde{a}\left(\delta \eta ; u^{m+\theta}-u_{h}^{m+\theta}, v_{h}\right) .
\end{aligned}
$$

Proof. We proceed as in the proof of Lemma 5.1 in [25]. We first recall that $\widetilde{u} \in$ $C^{1}([0, T] ; H)$ implies

$$
\left(\dot{\vec{u}}^{m+\theta}, v\right)+a\left(\widetilde{u}^{m+\theta}, v\right)=-\widetilde{a}\left(\delta \eta ; u^{m+\theta}, v\right), \quad \forall v \in V .
$$


Inserting the definition of $\xi_{h}^{m}$ into the left hand side of equation (27) yields

$$
\begin{aligned}
& (\Delta t)^{-1}\left(\xi_{h}^{m+1}-\xi_{h}^{m}, v_{h}\right)+a\left(\xi_{h}^{m+\theta}, v_{h}\right) \\
& =\left((\Delta t)^{-1}\left[\left(P_{h} \widetilde{u}^{m+1}-\widetilde{u}_{h}^{m+1}\right)-\left(P_{h} \widetilde{u}^{m}-\widetilde{u}_{h}^{m}\right)\right], v_{h}\right)+a\left(P_{h} \widetilde{u}^{m+\theta}, v_{h}\right)-a\left(\widetilde{u}_{h}^{m+\theta}, v_{h}\right) \\
& =\left((\Delta t)^{-1}\left(P_{h} \widetilde{u}^{m+1}-P_{h} \widetilde{u}^{m}\right), v_{h}\right)+a\left(P_{h} \widetilde{u}^{m+\theta}, v_{h}\right)-\left((\Delta t)^{-1}\left(\widetilde{u}_{h}^{m+1}-\widetilde{u}_{h}^{m}\right), v_{h}\right) \\
& \quad-a\left(\widetilde{u}_{h}^{m+\theta}, v_{h}\right) \\
& \quad \stackrel{(22)}{=}\left((\Delta t)^{-1}\left(P_{h} \widetilde{u}^{m+1}-P_{h} \widetilde{u}^{m}\right), v_{h}\right)+a\left(P_{h} \widetilde{u}^{m+\theta}, v_{h}\right) \\
& \quad+\widetilde{a}\left(\delta \eta ; u^{m+\theta}-u^{m+\theta}+u_{h}^{m+\theta}, v_{h}\right) \\
& \quad \stackrel{(28)}{=}\left((\Delta t)^{-1}\left(P_{h} \widetilde{u}^{m+1}-P_{h} \widetilde{u}^{m}\right)-\dot{\tilde{u}}^{m+\theta}, v_{h}\right)+a\left(P_{h} \widetilde{u}^{m+\theta}-\widetilde{u}^{m+\theta}, v_{h}\right) \\
& \quad-\widetilde{a}\left(\delta \eta ; u^{m+\theta}-u_{h}^{m+\theta}, v_{h}\right) .
\end{aligned}
$$

By stability (26) we have

Corollary A.4. Let $\widetilde{u} \in C^{1}([0, T] ; H)$. Then, under the assumptions of Lemma A.1,

$$
\left\|\xi_{h}^{M}\right\|^{2}+C_{1} \Delta t \sum_{m=0}^{M-1}\left\|\xi_{h}^{m+\theta}\right\|_{a}^{2} \leq\left\|\xi_{h}^{0}\right\|^{2}+C_{2} \Delta t \sum_{m=0}^{M-1}\left\|r^{m+\theta}\right\|_{*}^{2}
$$

We estimate the quantities $\left\|r_{j}^{m}\right\|_{*}, j=1, \ldots, 4$. For $j=1,2,3$ the estimates can be found in [25, Section 5]. For clarity, we restate them:

$$
\begin{aligned}
& \left\|r_{1}^{m}\right\|_{*} \leq C \begin{cases}(\Delta t)^{\frac{1}{2}}\left(\int_{t_{m}}^{t_{m+1}}\|\ddot{\widetilde{u}}(\tau)\|_{*}^{2} \mathrm{~d} \tau\right)^{\frac{1}{2}}, \quad \theta \in[0,1], \\
(\Delta t)^{\frac{3}{2}}\left(\int_{t_{m}}^{t_{m+1}}\|\dddot{\widetilde{u}}(\tau)\|_{*}^{2} \mathrm{~d} \tau\right)^{\frac{1}{2}}, \quad \theta=\frac{1}{2},\end{cases} \\
& \left\|r_{2}^{m}\right\|_{*} \leq C(\Delta t)^{-\frac{1}{2}} h^{s-r}\left(\int_{t_{m}}^{t_{m+1}}\|\dot{\widetilde{u}}(\tau)\|_{\mathcal{H}^{s-r}}^{2} \mathrm{~d} \tau\right)^{\frac{1}{2}}, \\
& \left\|r_{3}^{m}\right\|_{*} \leq C h^{s-r}\left\|\widetilde{u}^{m+\theta}\right\|_{\mathcal{H}^{s}}
\end{aligned}
$$

To estimate $r_{4}^{m}$, we assume that the bilinear form $\widetilde{a}(\delta \eta, \cdot, \cdot)$ is continuous on $V \times V$. Hence

$$
\left|\left(r_{4}^{m}, v_{h}\right)\right| \leq \widetilde{\alpha}\left\|u^{m+\theta}-u_{h}^{m+\theta}\right\|_{a}\left\|v_{h}\right\|_{a} .
$$

We obtain

Lemma A.5. Assume $\widetilde{u}(x, t)$ is sufficiently smooth in $[0, T] \times \bar{G}$ and assume that $\widetilde{a}(\delta \eta ; \cdot, \cdot)$ is continuous on $V \times V$. Then

$$
\left\|r^{m}\right\|_{*} \leq \sum_{j=1}^{3}\left\|r_{j}^{m}\right\|_{*}+C\left\|u^{m+\theta}-u_{h}^{m+\theta}\right\|_{a},
$$

with $\left\|r_{j}^{m}\right\|_{*}$ given by (30)-(32).

We are now able to prove Theorem 2.

Proof of Theorem 2. By definition $e_{h}^{m}=\eta^{m}+\xi_{h}^{m}, m=0,1, \ldots, M-1$. Hence

$\left\|e_{h}^{M}\right\|^{2}+\Delta t \sum_{m=0}^{M-1}\left\|e_{h}^{m+\theta}\right\|_{a}^{2} \leq 2\left(\left\|\eta^{M}\right\|^{2}+\Delta t \sum_{m=0}^{M-1}\left\|\eta^{m+\theta}\right\|_{a}^{2}\right)+2\left(\left\|\xi_{h}^{M}\right\|^{2}+\Delta t \sum_{m=0}^{M-1}\left\|\xi_{h}^{m+\theta}\right\|_{a}^{2}\right)$ 
The first term can be estimated by the approximation property (15). For the second term we have by the stability (29)

$$
\left\|\xi_{h}^{M}\right\|^{2}+\Delta t \sum_{m=0}^{M-1}\left\|\xi_{h}^{m+\theta}\right\|_{a}^{2} \leq\left\|\xi_{h}^{0}\right\|^{2}+C_{2} \Delta t \sum_{m=0}^{M-1}\left\|r^{m+\theta}\right\|_{*}^{2} .
$$

Using the estimates for $\left\|r^{m+\theta}\right\|_{*}$ of Lemma A.5 and the convergence result Lemma A.2 for the sequence $\left\{u_{h}^{m}\right\}$ finishes the proof.

A.2. Proof of Theorem 3. We estimate the error $e_{h}^{m}:=D^{\alpha} u^{m}-D_{h}^{\alpha} u_{h}^{m}$. We consider the splitting

$$
e_{h}^{m}=\underbrace{\left(D^{\alpha} u^{m}-P_{h} D^{\alpha} u^{m}\right)}_{\eta^{m}}+\underbrace{\left(P_{h} D^{\alpha} u^{m}-P_{h} D_{h}^{\alpha} u^{m}\right)}_{\nu_{h}^{m}}+\underbrace{\left(P_{h} D_{h}^{\alpha} u^{m}-D_{h}^{\alpha} u_{h}^{m}\right)}_{\xi_{h, \alpha}^{m}} .
$$

For $D_{h}^{\alpha} u^{m}$ and $D_{h}^{\alpha} u_{h}^{m}$ to be well defined, we extend $u^{m}$ and $u_{h}^{m}$ by zero to all of $\mathbb{R}^{d}$.

If $u^{m}$ is sufficiently smooth such that $D^{\alpha} u^{m} \in \mathcal{H}^{s}, \eta^{m}$ can be estimated using the approximation property (15). If we further assume that the projector $P_{h}: V \rightarrow V_{h}$ is uniformly stable (i.e. there exists a constant $C$ independent of $h$ such that $\left\|P_{h} v\right\|_{V} \leq$ $C\|v\|_{V}$ ), the term $\nu_{h}^{m}$ can be estimated using the approximation property (24) of $D_{h}^{\alpha}$. It therefore remains to estimate $\xi_{h, \alpha}^{m}$.

We shall need the following subspace of $V_{h}$. For $G_{0} \subset \subset G$ we denote by $V_{h}\left(G_{0}\right)$ the space

$$
V_{h}\left(G_{0}\right)=\left\{v \in V_{h} \mid \operatorname{supp} v \subseteq \bar{G}_{0}\right\} \subset V_{h} .
$$

We may assume that $G_{0}$ is such that $\varphi \in V_{h}\left(G_{0}\right)$ implies $D_{h}^{\alpha} \varphi \in V_{h}$. It is obviously sufficient to consider $\xi_{h, \alpha}^{m}:=P_{h} \partial_{h}^{\alpha} u^{m}-\partial_{h}^{\alpha} u_{h}^{m}$.

Lemma A.6. Assume $u \in C^{1}([0, T] ; H)$. Assume that the operators $P_{h}$ and $\partial_{h}^{\alpha}$ commute. Then $\left\{\xi_{h, \alpha}^{m}\right\}_{m=0}^{M-1}$ satisfy for any $\alpha \in \mathbb{N}_{0}^{d},|\alpha|=n \geq 0$ the $\theta$-scheme: Given $\xi_{h, \alpha}^{0}=$ $P_{h} \partial_{h}^{\alpha} u_{0}-\partial_{h}^{\alpha} u_{h}^{0}$, for $m=0,1, \ldots, M-1$

$$
(\Delta t)^{-1}\left(\xi_{h, \alpha}^{m+1}-\xi_{h, \alpha}^{m}, v_{h}\right)+a\left(\xi_{h, \alpha}^{m+\theta}, v_{h}\right)=\left(r^{m}, v_{h}\right), \quad \forall v_{h} \in V_{h}\left(G_{0}\right),
$$

with weak residual $r^{m}: V_{h} \rightarrow \mathbb{R}$ given by

where

$$
r^{m}=\sum_{j=1}^{4} r_{j}^{m}
$$

$$
\begin{aligned}
&\left(r_{1}^{m}, v_{h}\right):=(-1)^{|\alpha|}\left((\Delta t)^{-1}\left(u^{m+1}-u^{m}\right)-\dot{u}^{m+\theta}, \partial_{h}^{\alpha} v_{h}\right), \\
&\left(r_{2}^{m}, v_{h}\right):=(-1)^{|\alpha|}\left((\Delta t)^{-1}\left(P_{h} u^{m+1}-P_{h} u^{m}\right)-(\Delta t)^{-1}\left(u^{m+1}-u^{m}\right), \partial_{h}^{\alpha} v_{h}\right), \\
&\left(r_{3}^{m}, v_{h}\right):=(-1)^{|\alpha|} a\left(P_{h} u^{m+\theta}-u^{m+\theta}, \partial_{h}^{\alpha} v_{h}\right), \\
&\left(r_{4}^{m}, v_{h}\right):=-\int_{G} \sum_{\beta<\alpha} C_{\alpha, \beta}\left\{\sum_{i, j=1}^{d}\left(\partial_{h}^{\alpha-\beta} \mathcal{Q}_{i j}\right)\left(T_{h}^{\alpha-\beta} \partial_{h}^{\beta} D_{i}\left(P_{h} u^{m+\theta}-u_{h}^{m+\theta}\right)\right) D_{j} v_{h}\right. \\
&\left.\quad+\sum_{i=1}^{d}\left(\partial_{h}^{\alpha-\beta} b_{i}\right)\left(T_{h}^{\alpha-\beta} \partial_{h}^{\beta} D_{i}\left(P_{h} u^{m+\theta}-u_{h}^{m+\theta}\right)\right) v_{h}\right\} \mathrm{d} x
\end{aligned}
$$

with $C_{\alpha, \beta}:=\left(\begin{array}{c}\alpha \\ \alpha-\beta\end{array}\right)$. 
Proof. Recall that $u \in C^{1}([0, T], H)$ implies

$$
\left(\dot{u}^{m+\theta}, v\right)+a\left(u^{m+\theta}, v\right)=\left(f^{m+\theta}, v\right), \quad \forall v \in V .
$$

Let $v_{h} \in V_{h}\left(G_{0}\right)$. Inserting $\xi_{h, \alpha}^{m}$ in the $\theta$-scheme yields

$$
\begin{aligned}
(\Delta t)^{-1}\left(\xi_{h, \alpha}^{m+1}-\xi_{h, \alpha}^{m}, v_{h}\right)+a\left(\xi_{h, \alpha}^{m+\theta}, v_{h}\right) & \\
= & \left((\Delta t)^{-1}\left(P_{h} \partial_{h}^{\alpha} u^{m+1}-P_{h} \partial_{h}^{\alpha} u^{m}\right), v_{h}\right)+a\left(P_{h} \partial_{h}^{\alpha} u^{m+\theta}, v_{h}\right) \\
& -\left\{(\Delta t)^{-1}\left(\left(\partial_{h}^{\alpha} u_{h}^{m+1}-\partial_{h}^{\alpha} u_{h}^{m}\right), v_{h}\right)+a\left(\partial_{h}^{\alpha} u_{h}^{m+\theta}, v_{h}\right)\right\} .
\end{aligned}
$$

By the discrete Leibniz rule, we have

$$
\begin{aligned}
& (\Delta t)^{-1}\left(\left(\partial_{h}^{\alpha} u_{h}^{m+1}-\partial_{h}^{\alpha} u_{h}^{m}\right), v_{h}\right)+a\left(\partial_{h}^{\alpha} u_{h}^{m+\theta}, v_{h}\right) \\
& \quad=(-1)^{|\alpha|}\left((\Delta t)^{-1}\left(u_{h}^{m+1}-u_{h}^{m}\right), \partial_{h}^{\alpha} v_{h}\right)+(-1)^{|\alpha|} a\left(u_{h}^{m+\theta}, \partial_{h}^{\alpha} v_{h}\right)+R_{h}^{\alpha}\left(u_{h}^{m+\theta}\right) \\
& \quad \stackrel{(16)}{=}(-1)^{|\alpha|}\left(f^{m+\theta}, \partial_{h}^{\alpha} v_{h}\right)+R_{h}^{\alpha}\left(u_{h}^{m+\theta}\right) \\
& \quad \stackrel{(34)}{=}(-1)^{|\alpha|}\left(\dot{u}^{m+\theta}, \partial_{h}^{\alpha} v_{h}\right)+(-1)^{|\alpha|} a\left(u^{m+\theta}, \partial_{h}^{\alpha} v_{h}\right)+R_{h}^{\alpha}\left(u_{h}^{m+\theta}\right) .
\end{aligned}
$$

Here, we denote by $R_{h}^{\alpha}\left(u_{h}^{m+\theta}\right)$ the residual term

$$
\begin{aligned}
R_{h}^{\alpha}\left(u_{h}^{m+\theta}\right):=- & \int_{G} \sum_{\beta<\alpha} C_{\alpha, \beta}\left\{\sum_{i, j=1}^{d}\left(\partial_{h}^{\alpha-\beta} \mathcal{Q}_{i j}\right)\left(T_{h}^{\alpha-\beta} \partial_{h}^{\beta} D_{i} u_{h}^{m+\theta}\right) D_{j} v_{h}\right. \\
& \left.\left.+\sum_{i=1}^{d}\left(\partial_{h}^{\alpha-\beta} b_{i}\right)\left(T_{h}^{\alpha-\beta} \partial_{h}^{\beta} D_{i} u_{h}^{m+\theta}\right)\right) v_{h}\right\} \mathrm{d} x .
\end{aligned}
$$

Utilizing once more the discrete Leibniz rule and the fact that the operators $P_{h}$ and $\partial_{h}^{\alpha}$ commute yields

$$
\begin{aligned}
& \left((\Delta t)^{-1}\left(P_{h} \partial_{h}^{\alpha} u^{m+1}-P_{h} \partial_{h}^{\alpha} u^{m}\right), v_{h}\right)+a\left(P_{h} \partial_{h}^{\alpha} u^{m+\theta}, v_{h}\right) \\
& \quad=(-1)^{|\alpha|}\left(P_{h} u^{m+1}-P_{h} u^{m}, \partial_{h}^{\alpha} v_{h}\right)+(-1)^{|\alpha|} a\left(P_{h} u^{m+\theta}, \partial_{h}^{\alpha} v_{h}\right)+R_{h}^{\alpha}\left(P_{h} u^{m+\theta}\right) .
\end{aligned}
$$

The representation of $r^{m}$ in (33) is now obvious.

REMARK 3. Note that the residual $r_{4}^{m}$ in the Lemma A.6 satisfies $r_{4}^{m}=0$ if the coefficients $\mathcal{Q}, b$ in (3) are constant, as it is the case for the multidimensional Lévy model in Example 1.

By the stability result (Lemma A.1) we obtain the stability estimate for $\xi_{h, \alpha}^{m}$ in (33)

$$
\left\|\xi_{h, \alpha}^{M}\right\|^{2}+C_{1} \Delta t \sum_{m=0}^{M-1}\left\|\xi_{h, \alpha}^{m+\theta}\right\|_{a}^{2} \leq\left\|\xi_{h, \alpha}^{0}\right\|^{2}+C_{2} \Delta t \sum_{m=0}^{M-1}\left\|r^{m+\theta}\right\|_{*}^{2} .
$$

We estimate the residuals $\left\|r_{j}^{m}\right\|_{*}$. For $j=1,2,3$ these are the same as Lemma A.5 (with $u$ replacing $\widetilde{u}$, see also [25, Section 5]. To estimate $\left\|r_{4}^{m}\right\|$, we use again that the operators $P_{h}$ and $\partial_{h}^{\alpha}$ commute. We find

$$
\left\|r_{4}^{m}\right\|_{*} \leq C \sum_{\beta<\alpha}\left\|\partial_{h}^{\beta}\left(P_{h} u^{m+\theta}-u_{h}^{m+\theta}\right)\right\|_{a}=C \sum_{\beta<\alpha}\left\|\xi_{h, \beta}^{m+\theta}\right\|_{a} .
$$


Rewriting the estimate (35) shows that for any $\alpha \in \mathbb{N}_{0}^{d}$,

$$
\begin{aligned}
& \left\|\xi_{h, \alpha}^{M}\right\|^{2}+C_{1} \Delta t \sum_{m=0}^{M-1}\left\|\xi_{h, \alpha}^{m+\theta}\right\|_{a}^{2} \\
& \quad \leq\left\|\xi_{h, \alpha}^{0}\right\|^{2}+4 C_{2} \Delta t \sum_{m=0}^{M-1} \sum_{j=1}^{3}\left\|r_{j}^{m+\theta}\right\|_{a}^{2}+C \Delta t \sum_{m=0}^{M-1} \sum_{\beta<\alpha}\left\|\xi_{h, \beta}^{m+\theta}\right\|_{a}^{2} .
\end{aligned}
$$

Since (36) holds for an arbitrary (but fixed) $\alpha \in \mathbb{N}_{0}^{d}$, we may iterate the inequality until $\beta=0$ to obtain

$$
\begin{aligned}
\left\|\xi_{h, \alpha}^{M}\right\|^{2}+C_{1} \Delta t \sum_{m=0}^{M-1}\left\|\xi_{h, \alpha}^{m+\theta}\right\|_{a}^{2} \leq & C(\alpha) \Delta t \sum_{m=0}^{M-1}\left\|r_{1}^{m+\theta}\right\|_{a}^{2}+\left\|r_{2}^{m+\theta}\right\|_{a}^{2}+\left\|r_{3}^{m+\theta}\right\|_{a}^{2}+\left\|\xi_{h, 0}^{m+\theta}\right\|_{a}^{2} \\
& +C(\alpha) \sum_{\beta \leq \alpha}\left\|\xi_{h, \beta}^{0}\right\|^{2}
\end{aligned}
$$

Proof of Theorem 3. We have, for $e_{h}^{m}=D^{\alpha} u^{m}-D_{h}^{\alpha} u_{h}^{m}$ and $M \geq 1$,

$$
\begin{aligned}
& \left\|e_{h}^{M}\right\|^{2}+\Delta t \sum_{m=0}^{M-1}\left\|e_{h}^{m+\theta}\right\|_{a}^{2} \\
& \leq 3\left\{\left\|\eta^{M}\right\|^{2}+\Delta t \sum_{m=0}^{M-1}\left\|\eta^{m+\theta}\right\|_{a}^{2}+\left\|\nu_{h}^{M}\right\|^{2}+\Delta t \sum_{m=0}^{M-1}\left\|\nu_{h}^{m+\theta}\right\|_{a}^{2}+\left\|\xi_{h, \alpha}^{M}\right\|^{2}\right. \\
& \left.\quad+\Delta t \sum_{m=0}^{M-1}\left\|\xi_{h, \alpha}^{m+\theta}\right\|_{a}^{2}\right\} .
\end{aligned}
$$

If $D^{\alpha} u(t) \in \mathcal{H}^{s}$ for $t \in[0, T]$, the first term can be estimated with the approximation property (15). The second term is estimated using the uniform stability of the projector $P_{h}$ and the approximation property $(24)$ of $D_{h}^{\alpha}$ (provided $u(t) \in \mathcal{H}^{s+r+n}$ for $t \in[0, T]$ ). For the last term we have by $(37)$ and the fact that $\Delta t\left\|\xi_{h, 0}^{m+\theta}\right\|_{a}$ can be estimated also by the quantities $\left\|r_{j}^{m+\theta}\right\|_{*}, j=1,2,3$,

$$
\left\|\xi_{h, \alpha}^{M}\right\|^{2}+\Delta t \sum_{m=0}^{M-1}\left\|\xi_{h, \alpha}^{m+\theta}\right\|_{a}^{2} \leq C \sum_{\beta \leq \alpha}\left\|\xi_{h, \beta}^{0}\right\|^{2}+C \Delta t \sum_{m=0}^{M-1} \sum_{j=1}^{3}\left\|r_{j}^{m+\theta}\right\|_{a}^{2}
$$

Now conclude by using (30)-(32) to bound $\left\|r_{j}^{m+\theta}\right\|_{*}$ (replacing $\widetilde{u}$ by $u$ ), the quasioptimality of $\partial_{h}^{\beta} u_{h}^{0}$ and the approximation property (15) to estimate $\left\|\xi_{h, \beta}^{0}\right\|$.

\section{References}

[1] Y. Achdou and O. Pironneau, Computational Methods for Option Pricing, Frontiers in Applied Mathematics 30, SIAM, Philadelphia, PA, 2005.

[2] G. A. Baker, J. H. Bramble, and V. Thomée, Single step Galerkin approximations for parabolic problems, Math. Comp. 31 (1977), 818-847. 
[3] O. E. Barndorff-Nielsen and N. Shephard, Non-Gaussian Ornstein-Uhlenbeck-based models and some of their uses in financial economics, J. R. Stat. Soc. Ser. B Stat. Methodol. 63 (2001), 167-241.

[4] F. Black and M. Scholes, The pricing of options and corporate liabilities, J. Polit. Econ. 81 (1973), 637-654.

[5] J. H. Bramble and A. H. Schatz, Higher order local accuracy by averaging in the finite element method, Math. Comp. 31 (1977), 94-111.

[6] J. H. Bramble, J. A. Nitsche, and A. H. Schatz, Maximum-norm interior estimates for Ritz-Galerkin methods, Math. Comp. 29 (1975), 677-688.

[7] J. H. Bramble, A. H. Schatz, V. Thomée, and L. B. Wahlbin, Some convergence estimates for semidiscrete Galerkin type approximations for parabolic equations, SIAM J. Numer. Anal. 14 (1977), 218-241.

[8] M. Broadie and P. Glasserman, Estimating security price derivatives using simulation, Management Sci. 42 (1996), 269-285.

[9] P. Carr, H. Geman, D. B. Madan, and M. Yor, The fine structure of asset returns: an empirical investigation, J. Business 75 (2002), 305-332.

[10] P. Carr, H. Geman, D. B. Madan, and M. Yor, Self-decomposability and option pricing, Math. Finance 17 (2007), 31-57.

[11] W. Farkas, N. Reich, and C. Schwab, Anisotropic stable Lévy copula processes - analytical and numerical aspects, Math. Models and Methods in Appl. Sciences 17 (2007), 1405-1443.

[12] J. P. Fouque, G. Papanicolaou, and K. R. Sircar, Derivatives in Financial Markets with Stochastic Volatility, Cambridge University Press, Cambridge, 2000.

[13] E. Fournie, J.-M. Lasry, J. Lebuchoux, and P.-L. Lions, Applications of Malliavin calculus to monte carlo methods in finance II, Finance Stochastics 5 (2001), 201-236.

[14] S. L. Heston, A closed-form solution for options with stochastic volatility, with applications to bond and currency options, The Review of Financial Studies 6 (1993), 327-343.

[15] L. Jiang, Mathematical Modeling and Methods of Option Pricing, World Scientific, River Edge, NJ, 2005.

[16] J. Kallsen and P. Tankov, Characterization of dependence of multidimensional Lévy processes using Lévy copulas, J. Multivar. Anal. 97 (2006), 1551-1572.

[17] D. B. Madan, P. Carr, and E. Chang, The variance gamma process and option pricing, European Finance Review 2 (1998), 79-105.

[18] A.-M. Matache, T. von Petersdorff, and C. Schwab, Fast deterministic pricing of options on Lévy driven assets, M2AN Math. Model. Numer. Anal. 38 (2004), 37-71.

[19] E. Nicolato and E. Venardos, Option pricing in stochastic volatility models of the OrnsteinUhlenbeck type, Math. Finance 13 (2003), 445-466.

[20] J. A. Nitsche and A. H. Schatz, Interior estimates for Ritz-Galerkin methods, Math. Comp. 28 (1974), 937-958.

[21] O. Reiss and U. Wystup, Efficient computation of option price sensitivities using homogeneity and other tricks, J. Deriv. 9 (2001), 41-53.

[22] P. Tankov, Dependence structure of Lévy processes with applications to risk management, Rapport Interne No. 502, CMAPX École Polytechnique, Mars 2003.

[23] V. Thomée, High order local approximations to derivatives in the finite element method, Math. Comp. 31 (1977), 652-660.

[24] V. Thomée, Some interior estimates for semidiscrete Galerkin approximations for parabolic equations, Math. Comp. 33 (1979), 37-62. 
[25] T. von Petersdorff and C. Schwab, Wavelet discretizations of parabolic integrodifferential equations, SIAM J. Numer. Anal. 41 (2003), 159-180.

[26] T. von Petersdorff and C. Schwab, Numerical solution of parabolic equations in high dimensions, M2AN Math. Model. Numer. Anal. 38 (2004), 93-127. 